\title{
Prehemodialysis arteriovenous access creation is associated with better cardiovascular outcomes in patients receiving hemodialysis: A population-based cohort study
}

\author{
Cheng-Chieh Yen ${ }^{1}{ }^{\text {, }}$ Mei-Yin Liu ${ }^{2}$, Po-Wei Chen ${ }^{3}$ ， Peir-Haur Hung ${ }^{1}$, Tse-Hsuan Su ${ }^{\text {Corresp., }}{ }^{4}$, Yueh-Han Hsu \\ Corresp. $1,5,6$ \\ 1 Division of Nephrology, Department of Internal Medicine, Ditmansion Medical Foundation Chia-Yi Christian Hospital, Chia-Yi City, Taiwan \\ 2 Health Center, Municipal Jingliau Junior High School, Tainan City, Taiwan \\ 3 Division of Cardiology, Department of Internal Medicine, National Cheng Kung University Hospital, College of Medicine, National Cheng Kung University, \\ Tainan City, Taiwan \\ 4 Department of Emergency Medicine, Chang Gung Memorial Hospital Linkou, Taoyuan City, Taiwan \\ 5 Department of Medical Research, China Medical University Hospital, China Medical University, Taichung City, Taiwan \\ 6 Department of Nursing, Min-Hwei College of Health Care Management, Tainan City, Taiwan \\ Corresponding Authors: Tse-Hsuan Su, Yueh-Han Hsu \\ Email address: Hsuan.ths@gmail.com, cych07023@gmail.com
}

Background. Cardiovascular disease contributes to nearly half of the mortalities in patients with endstage renal disease. Patients who received prehemodialysis arteriovenous access (pre-HD AVA) creation had divergent cardiovascular outcomes.

Methods. We conducted a population-based cohort study by recruiting incident patients receiving HD from 2001 to 2012 from the Taiwan National Health Insurance Research Database. Patients' characteristics, comorbidities, and medicines were analyzed. The primary outcome of interest was major adverse cardiovascular events (MACEs), defined as hospitalization due to acute myocardial infarction, stroke, or congestive heart failure (CHF) occurring within the first year of HD. Secondary outcomes included MACE-related mortality and all-cause mortality in the same follow-up period.

Results. The patients in the pre-HD AVA group were younger, had a lower burden of underlying diseases, were more likely to use erythropoiesis-stimulating agents but less likely to use renin-angiotensin-aldosterone system blockers. The patients with pre-HD AVA creation had a marginally lower rate of MACEs but a significant 35\% lower rate of CHF hospitalization than those without creation (adjusted hazard ratio: 0.65 [95\% confidence interval: 0.48-0.88]). In addition, the pre-HD AVA group exhibited an insignificantly lower rate of MACE-related mortality but a significantly $52 \%$ lower rate of allcause mortality than the non-pre-HD AVA group (adjusted hazard ratio: 0.48 [95\% confidence interval: 0.39-0.59]). Sensitivity analyses obtained consistent results.

Conclusions. Pre-HD AVA creation is associated with a lower rate of CHF hospitalization and overall death in the first year of dialysis. 


\section{Prehemodialysis arteriovenous access creation is associated with}

\section{2 better cardiovascular outcomes in patients receiving hemodialysis:}

\section{A population-based cohort study}

4

5

6 Cheng-Chieh Yen ${ }^{1}$, Mei-Yin Liu ${ }^{2}$, Po-Wei Chen ${ }^{3}$, Peir-Haur Hung ${ }^{1}$, Tse-Hsuan Su${ }^{4}$, Yueh-Han $7 \mathrm{Hsu}^{1,5,6}$

$8{ }^{1}$ Division of Nephrology, Department of Internal Medicine, Ditmansion Medical Foundation

9 Chia-Yi Christian Hospital, Chia-Yi City, Taiwan

$10{ }^{2}$ Health Center, Municipal Jingliau Junior High School, Tainan City, Taiwan

11

12

13

${ }^{3}$ Division of Cardiology, Department of Internal Medicine, National Cheng Kung University Hospital, College of Medicine, National Cheng Kung University, Tainan

${ }^{4}$ Department of Emergency Medicine, Chang Gung Memorial Hospital Linkou, Taoyuan City, Taiwan

${ }^{5}$ Department of Medical Research, China Medical University Hospital, China Medical University, Taichung City, Taiwan

${ }^{6}$ Department of Nursing, Min-Hwei College of Health Care Management, Tainan City, Taiwan

\section{Corresponding Author}

Tse-Hsuan Su, M.D., M.S.and Yueh-Han Hsu

Department of Emergency Medicine, Chang Gung Memorial Hospital Linkou, Taoyuan City, Taiwan

Division of Nephrology, Department of Internal Medicine, Ditmansion Medical Foundation Chia-Yi Christian Hospital, Chia-Yi City, Taiwan; Department of Medical Research, China Medical University Hospital, China Medical University, Taichung City, Taiwan; Department of Nursing, Min-Hwei College of Health Care Management, Tainan City, Taiwan Tel: +8863328 1200 ext. 3523

Fax: +88633287715

Email: Hsuan.ths@gmail.com; cych07023@gmail.com 


\section{Abstract}

Background. Cardiovascular disease contributes to nearly half of the mortalities in patients with end-stage renal disease. Patients who received prehemodialysis arteriovenous access (preHD AVA) creation had divergent cardiovascular outcomes.

Methods. We conducted a population-based cohort study by recruiting incident patients receiving HD from 2001 to 2012 from the Taiwan National Health Insurance Research Database. Patients' characteristics, comorbidities, and medicines were analyzed. The primary outcome of interest was major adverse cardiovascular events (MACEs), defined as hospitalization due to acute myocardial infarction, stroke, or congestive heart failure (CHF) occurring within the first year of HD. Secondary outcomes included MACE-related mortality and all-cause mortality in the same follow-up period.

Results. The patients in the pre-HD AVA group were younger, had a lower burden of underlying diseases, were more likely to use erythropoiesis-stimulating agents but less likely to use renin-angiotensin-aldosterone system blockers. The patients with pre-HD AVA creation had a marginally lower rate of MACEs but a significant 35\% lower rate of CHF hospitalization than those without creation (adjusted hazard ratio: 0.65 [95\% confidence interval: $0.48-0.88]$ ). In addition, the pre-HD AVA group exhibited an insignificantly lower rate of MACE-related mortality but a significantly $52 \%$ lower rate of all-cause mortality than the non-pre-HD AVA group (adjusted hazard ratio: 0.48 [95\% confidence interval: $0.39-0.59]$ ). Sensitivity analyses obtained consistent results.

Conclusions. Pre-HD AVA creation is associated with a lower rate of CHF hospitalization and overall death in the first year of dialysis. 


\section{Introduction}

53 End-stage renal disease (ESRD) has become a major public health issue because of its

54 prevalence in more than 2 million people worldwide and increasing incidence. Compared with 55 general cohorts, patients with ESRD have a higher relative risk of 5-year mortality(Robinson et 56 al. 2016). Among the major causes of mortality, cardiovascular (CV) disease contributes nearly 57 half of the events in this population(Collins et al. 2010). Therefore, identifying management of 58 CV complications is essential.

59 Pre-hemodialysis (pre-HD) care has been proved to ameliorate the outcomes of patients

60

61

62 63 with ESRD maintained on HD(Baek et al. 2015; Bradbury et al. 2007). Timely creation of arteriovenous access (AVA), such as native fistula or artificial graft, is one of the crucial methods of care planning. It prevents not only the complications from delayed dialysis but also catheter-related infectious events(Oliver et al. 2004). However, CV outcomes following pre-HD AVA surgery are currently divergent. Once pre-HD AVA is created, cardiac output increases and leads to functional and structural changes of the heart, lungs and vasculature(Guyton \& Sagawa 1961; Munclinger et al. 1987). London and colleagues reported that the arteriovenous shunt might result in chronic flow overload and cause cardiac hypertrophy(London et al. 1993). Nakhoul et al observed that nitric oxygen production was decreased in patients with arteriovenous fistula and contributed to pulmonary hypertension(Nakhoul et al. 2005). Korsheed and colleagues reported improved arterial stiffness, better ejection fraction, and lesser heart damage after native fistula creation(Korsheed et al. 2009; Korsheed et al. 2011). Variation in laboratory and imaging parameters makes it difficult to predict the clinical outcomes. Several small-scale studies have reported negative clinical CV results following fistula creation(MacRae et al. 2004; Reddy et al. 2017; Vizinho et al. 2014), while a national study using the United States Renal Data System showed that using pre-HD fistula was strongly associated with lower 
76 CV mortality(Wasse et al. 2008). At present, only a few large-scale studies have explored the

77 association between AVA creation and CV-related hospitalization.

78 Although ESRD is reported to have the highest prevalence in Taiwan compared with other

79 countries, the 5-year survival rate of patients with ESRD seems better in Taiwan(Robinson et al.

80 2016). The Taiwan pre-ESRD pay-for-performance program, involving education and promotion

81 of pre-HD AVA establishment, might have contributed to the higher survival rate(Lin et al.

82 2018). In Taiwan, more than half of the patients undergoing dialysis received access surgery

83 before their first dialysis session, and access creation had been completed in more than $80 \%$ of

84 them before their chronic dialysis sessions(Hsu et al. 2018). Our study investigated the

85 association between timing of AVA creation and CV outcomes in patients who underwent HD.

86 We hypothesized that pre-HD AVA creation improves the CV outcomes of patients undergoing

87 HD. 


\section{Materials and Methods}

89

90

91

92

93

94

95

96

97

98

99

100

101

102

103

104

105

106

107

108

109

110

111

Data source

We conducted a retrospective cohort study by using the Taiwan National Health Insurance

Research Database (NHIRD), which is a national population-based database, provided by Taiwan National Health Insurance (NHI). The NHI is a single-payer, universal and compulsory healthcare program initiated in 1995 and covers $99.9 \%$ of Taiwanese residents(National Health Insurance Administration 2014). In this study, we used a representative subset of 1 million persons randomly sampled from the 24 million beneficiaries from the Taiwan NHI between 2000 and 2013. No significant difference was observed between the subset and NHIRD in the distribution of sex, age, and average insured payroll-related amount. All identities in the NHIRD are encrypted to guarantee patient privacy. This study was approved by the Institutional Review Board of Ditmanson Medical Foundation Chia-Yi Christian Hospital in Taiwan (CYCH-IRB No. 2018054). Informed consent was waived owing to the absence of interference in decisionmaking processes of medical care.

\section{Study design, identification and grouping of study subjects}

We identified patients with chronic kidney disease (CKD) who began HD sessions during 2001 to 2012 by using the NHI procedure codes of receiving HD. The day of first HD session was employed as the index date. CKD was defined as patients receiving at least 2 outpatient diagnoses according to International Classification of Diseases, 9th Revision, Clinical Modification (ICD-9-CM) codes within the 1 year prior to the index date. Patients were excluded if they were aged $<20$ years, had ever received peritoneal dialysis, or had kidney transplantation before or during their first year of HD. We combined patients receiving native fistula and artificial graft for analysis because of their similar CV results(Ravani et al. 2013). Pre-HD AVA 
112 was defined as its creation date $\geqq 1$ month before the index date. Patients in whom AVA was

113 created $<1$ month prior to HD were excluded owing to their inappropriate access usage

114 according to the guidelines(2006; Ishani et al. 2014). We further excluded patients who received 115 implantation of HD catheters, namely tunneled and nontunneled catheters, before the index date.

117 Data and definitions of study variables

118 We analyzed the characteristics, comorbidities, and medicines of the included patients.

119 Owing to the NHI charged its beneficiaries different amounts of insurance premiums according 120 to their earnings, the socioeconomic status of patients was represented by their income, which 121 was obtained according to the average insured payroll-related amounts. Comorbidities were 122 defined as patients experiencing at least 1 hospitalization or 2 outpatient visits, which expressed 123 in terms of the corresponding ICD-9-CM codes of any of the following illnesses, within the 1 124 year prior to the index date: hypertension (HTN), ischemic heart disease (IHD), congestive heart 125 failure (CHF), cerebrovascular accident (CVA), peripheral vascular disease (PVD), dysrhythmia, 126 diabetes mellitus (DM), chronic obstructive pulmonary disease (COPD), peptic ulcer disease 127 (PUD), liver disease, cancer, and dementia. Additionally, to denote the disease burdens we 128 applied the Taiwan index, which is a comorbidity index for mortality prediction validated for 129 Taiwanese patients with incident HD(Chen et al. 2014). Medicine including erythropoiesis130 stimulating agent (ESA), antiplatelet agent, anticoagulant agent, angiotensin converting enzyme 131 inhibitor (ACEI), angiotensin II receptor blocker (ARB) and statin was defined as more than 2

132 prescriptions during ambulatory visits within the 1 year prior to the index date and was expressed 133 in terms of the anatomical therapeutic chemical classification system. 
135

136

137

138

139

140

141

142

143

144

145

146

147

148

149

150

151

152

153

154

155

156

157

158

\section{Outcomes of study subjects}

Primary outcome of the present study was major adverse cardiovascular events (MACEs), which was defined as ICD-9-CM-based hospitalization for acute myocardial infarction (AMI), CVA, or CHF that occurred within the 1 year after the first HD session. Secondary outcomes were all-cause mortality, MACE-related and bloodstream infection (BSI)-related mortality, which was defined as overall death and death resulting from MACE and BSI in corresponding ICD-9-CM codes, within the same follow-up period following the first dialysis. To validate the findings, we performed multiple sensitivity analyses including exclusion patients without AVA creation, exclusion of patients not receiving regular HD, and inclusion of patient receiving AVA creation within 1 month.

\section{Statistical analysis}

We compared patient characteristics, comorbidities, and medical prescriptions between the pre-HD AVA and non-pre-HD AVA groups. Continuous data were reported as a median or mean and were analyzed using the Mann-Whitney test or independent $t$ test as appropriate. Categorical data were reported as percentages and were analyzed using the Chi-squared test. Since management of chronic kidney disease changed over time, we included the year of starting HD as a time indicator. We constructed our propensity score model using variables related to patient characteristics, year of HD, comorbidities, and medical prescriptions, and calculated model using the logistic regression method. We performed 1:1 matching using the nearest neighbor algorithm without replacement and with a 0.05 caliper width to reduce imbalances between groups. After matching, we used absolute standardized differences (ASD) to evaluate the balance between groups. An ASD threshold of 10\% was used to delineate good and poor balance(Austin 2008).

We evaluated the cause-specific mortality and overall mortality over 1 year using weighted 
159 cumulative incidence curves considering competing risks of death. Event-time was measured 160 from the index date until the date of the event, 1 year after the index date, or the end of the study 161 (31/12/2013), whichever occurred earlier. We applied the Fine-Gray subdistributional hazard 162 models to calculate crude and adjusted hazard ratios (HRs). Robust sandwich variance was used 163 to correct the correlated data structure after matching(Austin 2014). Because the 1:1 propensity 164 score matching would reduce the sample size, we performed the inverse probability treatment 165 weighting (IPTW) of study subjects as another sensitivity analysis. Since extreme weights might 166 cause bias estimate in the marginal hazard ratio, we used IPTW in the subsample restricted to 167 patients with a propensity score between 0.1 and 0.9(Austin \& Stuart 2017). A 2-tailed $p$ value of $168<0.05$ indicated statistical significance. SAS version 9.4 (SAS Institute, Inc.) was used for 169 analyses. 


\section{Results}

171 Figure 1 illustrates the research design and sampling strategy. Overall, this study analyzed

1723147 patients - 837 patients (26.6\%) receiving pre-HD AVA creation and 2310 patients $(73.4 \%)$

173 not receiving pre-HD AVA creation.

174 The baseline characteristics of the recruited patients are presented in Table 1. The median

175 age of patients with pre-HD AVA creation was lower than that of those without (66 years vs 71

176 years, ASD: 29.9\%). No statistically significant difference was observed in terms of sex between

177 the 2 groups. Considering comorbidities, the patients in the pre-HD AVA group had lower

178 proportions of CVA, dysrhythmia, COPD, liver disease, and dementia than those in the non-pre-

179 HD AVA group. The groups exhibited similar proportions of patients with IHD, CHF, PVD, and

180 DM. The mean Taiwan index of pre-HD AVA group was significantly lower than that of the

181 non-pre-HD AVA group (5.55 \pm 4.0 vs $6.55 \pm 4.4$, ASD: 23\%). Additionally, more patients with

182 pre-HD AVA creation received ESAs than did those without pre-HD AVA creation $(81.72 \%$ vs

183 34.81\%, ASD: 108\%), whereas less patients with pre-HD AVA received ACEIs and ARBs than

184 did those without pre-HD AVA (60.57\% vs 68.35\%, ASD: 16.3\%). The prescription of

185 antiplatelet agent, anticoagulant agent or statin was similar among the groups.

186 Table 2 shows the primary and secondary outcomes of the pre-HD AVA and non-pre-HD

187 AVA groups. The patients with pre-HD AVA creation had a lower rate of MACEs during the

188 follow-up period than did those without pre-HD AVA creation (crude HR: 0.73 [95\% confidence

189 interval (CI): 0.6-0.89]), but the effect became nonsignificant after matching for age, sex, year of

190 HD, comorbidities, and medicine (adjusted HR: 0.89 [95\% CI: 0.71-1.11], $p=0.29$, Figure 2).

191 We further analyzed the MACEs separately and observed that patients with pre-HD AVA

192 creation had a 35\% lower CHF hospitalization rate after matching (adjusted HR: 0.65 [95\% CI:

$1930.48-0.88], p<0.01$, Figure 3). We examined CHF hospitalization rate in the propensity-score- 
194 matched groups, which showed a similar trend (adjusted HR: 0.63 [95\% CI: 0.46-0.87], $p<$ $1950.01)$.

196 Regarding secondary outcomes, we revealed that patients in the pre-HD AVA group had a $19752 \%$ lower rate of all-cause mortality (adjusted HR: 0.48 [95\% CI: $0.39-0.59], p<0.001$, Figure 198 4), a marginally lower rate of MACE-related mortality (adjusted HR: 0.7 [95\% CI: 0.45-1.08], $p$ $199=0.1$, Figure 5), and a 68\% lower rate of BSI-related mortality (adjusted HR: 0.32 [95\% CI: $2000.22-0.46], p<0.001$, Supplementary Figure 1) than those in the non-pre-HD AVA group. We 201 next examined the outcomes in the propensity-score-matched groups. The patients with pre-HD 202 AVA creation exhibited a 54\% lower rate of all-cause mortality (adjusted HR: 0.46 [95\% CI: $2030.36-0.59$ ], $p<0.001$ ), a 40\% lower rate of MACE-related mortality (adjusted HR: 0.60 [95\% CI: $2040.38-0.96], p<0.05$ ), and a 71\% lower rate of BSI-related mortality (adjusted HR: 0.29 [95\% 205 CI: 0.19-0.44], $p<0.01$ ) than those without pre-HD AVA creation. 206 Sensitivity analyses of the outcomes were presented in Table 3 . They revealed a 207 consistently lower CHF hospitalization rates, ranging from $29 \%$ to $43 \%$, of patients receiving 208 pre-HD AVA creation. In addition, they exhibited a consistently lower BSI-related mortality 209 rates of patients in the pre-HD AVA group. 


\section{Discussion}

211 In our nationally representative cohort, we observed that patients with pre-HD AVA

212 creation had a 35\% lower CHF hospitalization rate and 52\% lower all-cause mortality rate than

213 those without during the first year of HD — significant differences. Additionally, we disclosed a

214 marginally lower rate of MACEs and MACE-related mortality during the same follow-up period.

215 Pre-HD AVA creation might be associated with better CV outcomes within the first year of HD.

216 In this study, the patients with pre-HD AVA creation were younger, had a lesser burden of

217 comorbidities, had a higher percentage of ESAs but a lower percentage of ACEIs and ARBs

218 usage (Table 1). Age is a well-established factor affecting postsurgery prognosis. The

219 significantly lower Taiwan index of the pre-HD AVA group indicated the lower disease burden

220 of the patients in the pre-HD AVA group compared with the non-pre-HD AVA group. We also

221 observed that the patients without pre-HD AVA creation were significantly more likely to have

222 CVA, dysrhythmia, COPD, or liver disease than those with pre-HD AVA creation, and those

223 diseases might reflect higher neurologic, respiratory, and coagulatory risks during the operation.

224 In one previous study, patients with dementia had a greater risk of early death and fatal

225 complications postoperatively(Kassahun 2018). Thus, patients who were young or had fewer

226 comorbidities were willing to undergo pre-HD AVA surgery. Additionally, a different proportion

227 of patients receiving ESAs and renin-angiotensin-aldosterone system blockers might imply

228 more recruitment of pre-HD care, which promotes the possibility of dialysis access creation by

229 education(Ishani et al. 2014).

230 Our study revealed a significantly lower CHF hospitalization rate within the first year of

231 HD among the patients receiving pre-HD AVA creation (Figure 3). Consistent results were

232 obtained for the other matched models (Table 2) and sensitivity analyses (Table 3). Patients who

233 undergo AVA surgery before HD might avoid delayed HD, thus preventing exacerbated fluid 
234 overload and increased CHF risk. The increased cardiac preload after AVA surgery is

235 compensated by a corresponding decrease in peripheral vascular resistance following surgery(Ori

236 et al. 1996) and consequent fluid removal during HD sessions. The fluid status of most patients

237 undergoing HD has been proved to achieve a new balance shortly(Alkhouli et al. 2015; Dal

238 Canton et al. 1981). In the Dialysis Outcomes and Practice Patterns Study, Rayner and

239 colleagues observed a low flow rate of the fistula in Japanese patients (Rayner et al. 2003),

240 which might be related to low-caliber vessels in the Asian population. The degree of CV damage

241 due to blood volume following AVA creation might differ according to vascular characteristics.

242 Several studies have supported our findings of CV benefits following pre-HD AVA creation: Ori

243 et al conducted an echocardiographic study to observe cardiac performance before and after

244 AVA creation. A gentle volume overload developed postoperatively but was offset by decreased

245 vascular resistance. The shortening and ejection fractions of the left ventricle were improved 2

246 weeks after the AVA operation(Ori et al. 1996). Sandhu and colleagues observed that none of 17

247 patients receiving native fistula before HD developed CHF during the 6 weeks following surgery.

248 They concluded that the postoperative changes in cardiac index, stroke volume, and vascular

249 resistance were physically minimal and without extra loading of patients' hemodynamics(Sandhu

250 et al. 2004). Thus, CHF might not occur or worsen after AVA creation. Further investigation is

251 warranted to clarify the causality of AVA in CV outcomes.

252 Some studies have obtained contrasting results from ours: MacRae et al noted that a patient

253 undergoing HD developed cardiac failure under a high-flow arteriovenous fistula and concluded

254 that the high fistula flow caused myocardium decompensation with a decline in the ejection

255 fraction(MacRae et al. 2004). Other studies have adopted an opposite viewpoint on ejection

256 fraction alteration following AVA creation(Iwashima et al. 2002; Korsheed et al. 2011; Ori et al.

257 1996). Vizinho and colleagues reported that pre-HD AVA creation was associated with a 
258 decrease in the subendocardial viability ratio, which predicted a poorer outcome regarding CV

259 hospitalization(Vizinho et al. 2014). Nevertheless, a small sample size and lack of CV

260 comorbidity adjustments limit the relevance of their speculation. Reddy et al traced CV changes

261 of patients following native shunt creation for 2.6 years and observed that remodeling and

262 dysfunction of the right ventricle developing after shunt operation and dialysis initiation caused

263 increased risks of CHF and death(Reddy et al. 2017). However, the absence of controls and

264 uncertainty in the effect of AVA and dialysis on cardiac dysfunction made the supposition

265 inconclusive. More large-scale and close-matching studies should be planned to confirm the

266 relationship.

267 Considering secondary outcomes, we evaluated the effect of pre-HD AVA creation on 268 overall mortality and disclosed a $52 \%$ lower rate of all-cause mortality in the pre-HD AVA 269 group (Table 2, Figure 4). In addition to CV disease, catheter-associated infectious disease, 270 mainly those transmitted through the bloodstream, is another major cause of mortality in patients 271 undergoing dialysis. We assumed that the lower all-cause mortality might have been related to 272 the $68 \%$ reduction in the rate of BSI-related mortality in the pre-HD AVA group (Table 2, 273 Supplementary Figure 1), which was due to lesser usage of HD catheters. Additionally, we also 274 observed that the patients with pre-HD AVA creation had an insignificantly lower rate of 275 MACE-related mortality compared with those without after propensity score matching (Table 2, 276 Figure 5). We believe that this type of mortality is mainly affected by the underlying diseases of 277 patients rather than AVA surgery. The literature has suggested that HTN, IHD, CHF, and DM 278 influence the CV mortality rate for patients undergoing HD(Banerjee et al. 2007; Lee et al. 2016; 279 Zoccali et al. 2005). Because our groups had similar distributions of these diseases, our finding 280 was in fair agreement with the literature.

281 This was a country-based study including all pre-HD patients underlined CKD matched for 
282 age, sex, income, year of HD, comorbidities, and associated medicine. In Taiwan, the NHI 283 Bureau has launched pay-for-performance program focusing on patients of glomerulus filtration 284 rate $<45 \mathrm{ml} / \mathrm{min} / 1.73 \mathrm{~m}^{2}$ from 2006 . It is incentive payment for medical institution if recruited 285 patients achieved the targets on blood pressure, glycated hemoglobin, nursing education, 286 nutrition consult, and so on. Patients with ESRD have received comprehensive access evaluation 287 by qualified nephrologists at outpatient clinics or during admissions. Most studies exploring the 288 effects of pre-HD AVA creation on CV outcomes compared associated parameters before and 289 after surgery(Dal Canton et al. 1981; Dundon et al. 2014; Iwashima et al. 2002; Korsheed et al. 2011; Munclinger et al. 1987; Ori et al. 2002; Ori et al. 1996; Reddy et al. 2017; Sandhu et al. 2004; Savage et al. 2002; Utescu et al. 2009; Vizinho et al. 2014). In addition, some compared the effects before dialysis initiation to exclude the impact of dialysis on $\mathrm{CV}$ performance(Dundon et al. 2014; Iwashima et al. 2002; Korsheed et al. 2011; Ori et al. 1996;

294 Savage et al. 2002). However, selection bias would have been unavoidable in these studies 295 because patients with pre-HD AVA creation tend to be compliant in medical practice, which 296 would affect their overall outcomes. Furthermore, excluding the dialysis effect appears 297 impractical considering the goal of AVA preparation. Moreover, dialysis is a well-known risk 298 factor of cardiac injury, and its vintage was positively associated with the degree of 299 injury(McIntyre 2009). Once the AVA was used for dialysis, evaluating the CV prognoses in combination with HD was difficult. We followed up for 1 year after HD initiation because we 301 assumed that the effect of pre-HD AVA creation would be offset by a longer period of HD. Our 302 study provides another perspective regarding evaluation of the benefits and hazards of pre-HD 303 AVA surgery.

304 This study had several limitations. First, the present study was an observational study, 305 which non-observed confounders might restrict the inference. Second, the NHIRD is an 
306 administrative database in which the identification of comorbidities is based solely on ICD-9-

$307 \mathrm{CM}$ codes rather than clinical criteria; misclassifications might thus have occurred, leading to

308 residual confounding. Additionally, we recruited our patients until the end of 2012 since the data

309 was valid until 31/12/2013 in LHID 2000. Third, patients could choose the preferred medical

310 providers for AVA creation or HD freely owing to high medical accessibility in Taiwan. It was

311 difficult to figure out the relationship between patients and medical providers, which have

312 influenced the timing of AVA creation. Fourth, the indications of CHF hospitalization are varied

313 among patients and medical facilities. However, the NHIRD does not provide objective

314 parameters of cardiac alteration, such as the level of natriuretic peptide, ejection fraction of

315 ventricles, or pulse wave velocity of vessels, which could support our findings. Fifth, our study

316 did not consider medications such as calcium channel blockers, beta blockers, or diuretics, which

317 have been shown to influence CV outcomes in patients undergoing $\mathrm{HD}$ (Georgianos \& Agarwal

318 2016; Karaboyas et al. 2018). Lastly, it was difficult to distinguish the absolute effect of pre-HD

319 AVA on CV outcomes in combination with personal compliance and dialysis factors affecting

320 the CV system. Integrated trials comprising data and imaging should be further conducted to

321 corroborate our results. 


\section{Conclusions}

323 In this population-based cohort study, patients with pre-HD AVA creation had a 35\% lower

324 CHF hospitalization rate and a 52\% lower all-cause mortality rate than those without pre-HD

325 AVA creation within the first year of HD. Marginal benefits were also observed in terms of

326 MACEs and MACE-related mortality during the same follow-up period. Pre-HD AVA creation

327 might be associated with better CV outcomes in the first year of HD and should be promoted in

328 the pre-HD care focusing on patients with late-stage CKD. 


\section{Acknowledgements}

330 We would like to thank Ching-Fang Tsai for the pretest of study design, and Professor

331 Chih-Cheng Hsu for his experienced dehortation and encouragement throughout the study. 
332

333

334

335

336

337

338

339

340

341

342

343

344

345

346

347

348

349

350

351

352

353

354

355

356

357

358

359

360

361

362

363

364

365

366

\section{References}

Introduction to the National Health Insurance Research Database (NHIRD), Taiwan. Available at http://nhird.nhri.org.tw/date 01 en.html (accessed 2017/11/01 2017).

2006. Clinical practice guidelines for vascular access. Am J Kidney Dis 48 Suppl 1:S248-273. 10.1053/j.ajkd.2006.04.040

Alkhouli M, Sandhu P, Boobes K, Hatahet K, Raza F, and Boobes Y. 2015. Cardiac complications of arteriovenous fistulas in patients with end-stage renal disease. Nefrologia 35:234-245. 10.1016/j.nefro.2015.03.001

Austin PC. 2008. A critical appraisal of propensity-score matching in the medical literature between 1996 and 2003. Stat Med 27:2037-2049. 10.1002/sim.3150

Austin PC. 2014. The use of propensity score methods with survival or time-to-event outcomes: reporting measures of effect similar to those used in randomized experiments. Stat Med 33:1242-1258. 10.1002/sim.5984

Austin PC, and Stuart EA. 2017. The performance of inverse probability of treatment weighting and full matching on the propensity score in the presence of model misspecification when estimating the effect of treatment on survival outcomes. Stat Methods Med Res 26:16541670. 10.1177/0962280215584401

Baek SH, Ahn S, Lee SW, Park YS, Kim S, Na KY, Chae DW, Kim S, and Chin HJ. 2015. Outcomes of predialysis nephrology care in elderly patients beginning to undergo dialysis. PLoS One 10:e0128715. 10.1371/journal.pone.0128715

Banerjee D, Ma JZ, Collins AJ, and Herzog CA. 2007. Long-term survival of incident hemodialysis patients who are hospitalized for congestive heart failure, pulmonary edema, or fluid overload. Clin J Am Soc Nephrol 2:1186-1190. 10.2215/cjn.01110307

Bradbury BD, Fissell RB, Albert JM, Anthony MS, Critchlow CW, Pisoni RL, Port FK, and Gillespie BW. 2007. Predictors of early mortality among incident US hemodialysis patients in the Dialysis Outcomes and Practice Patterns Study (DOPPS). Clin J Am Soc Nephrol 2:89-99. 10.2215/cjn.01170905

Chen JY, Tsai SH, Chuang PH, Chang CH, Chuang CL, Chen HL, and Chen PL. 2014. A comorbidity index for mortality prediction in Chinese patients with ESRD receiving hemodialysis. Clin J Am Soc Nephrol 9:513-519. 10.2215/cjn.03100313

Collins AJ, Foley RN, Herzog C, Chavers BM, Gilbertson D, Ishani A, Kasiske BL, Liu J, Mau LW, McBean M, Murray A, St Peter W, Guo H, Li Q, Li S, Li S, Peng Y, Qiu Y, Roberts T, Skeans M, Snyder J, Solid C, Wang C, Weinhandl E, Zaun D, Arko C, Chen SC, Dalleska F, Daniels F, Dunning S, Ebben J, Frazier E, Hanzlik C, Johnson R, Sheets D, Wang X, Forrest B, Constantini E, Everson S, Eggers PW, and Agodoa L. 2010. Excerpts from the US Renal 
367

368

369

370

371

372

373

374

375

376

377

378

379

380

381

382

383

384

385

386

387

388

389

390

391

392

393

394

395

396

397

398

399

400

401

402

Data System 2009 Annual Data Report. Am J Kidney Dis 55:S1-420, a426-427. 10.1053/j.ajkd.2009.10.009

Dal Canton A, Maione S, Russo D, Teti C, Serino C, Gallo R, and Andreucci VE. 1981. Echocardiographic detection of cardiac effects of arterio-venous dialysis fistula. Clin Exp Dial Apheresis 5:259-267.

Dundon BK, Torpey K, Nelson AJ, Wong DT, Duncan RF, Meredith IT, Faull RJ, Worthley SG, and Worthley MI. 2014. The deleterious effects of arteriovenous fistula-creation on the cardiovascular system: a longitudinal magnetic resonance imaging study. Int J Nephrol Renovasc Dis 7:337-345. 10.2147/ijnrd.s66390

Georgianos PI, and Agarwal R. 2016. Pharmacotherapy of Hypertension in Chronic Dialysis Patients. Clin J Am Soc Nephrol 11:2062-2075. 10.2215/cjn.00870116

Guyton AC, and Sagawa K. 1961. Compensations of cardiac output and other circulatory functions in areflex dogs with large A-V fistulas. Am J Physiol 200:1157-1163. 10.1152/ajplegacy.1961.200.6.1157

Hsu CC, Hsiung CA, Wu MS, Huang SJ, Lin YC, Hsu YH, and Chiu YW. 2018. 2017 Annual Report on Kidney Disease in Taiwan. Maoli County, Taiwan: National Health Research Institutes.

Ishani A, Gilbertson DT, Kim D, Bradbury BD, and Collins AJ. 2014. Predialysis care and dialysis outcomes in hemodialysis patients with a functioning fistula. Am J Nephrol 39:238247. 10.1159/000358843

Iwashima Y, Horio T, Takami Y, Inenaga T, Nishikimi T, Takishita S, and Kawano Y. 2002. Effects of the creation of arteriovenous fistula for hemodialysis on cardiac function and natriuretic peptide levels in CRF. Am J Kidney Dis 40:974-982. 10.1053/ajkd.2002.36329

Karaboyas A, Xu H, Morgenstern H, Locatelli F, Jadoul M, Nitta K, Dasgupta I, Tentori F, Port FK, and Robinson BM. 2018. DOPPS data suggest a possible survival benefit of renin angiotensin-aldosterone system inhibitors and other antihypertensive medications for hemodialysis patients. Kidney Int 94:589-598. 10.1016/j.kint.2018.03.013

Kassahun WT. 2018. The effects of pre-existing dementia on surgical outcomes in emergent and nonemergent general surgical procedures: assessing differences in surgical risk with dementia. BMC Geriatr 18:153. 10.1186/s12877-018-0844-x

Korsheed S, Burton JO, and McIntyre CW. 2009. Higher arteriovenous fistulae blood flows are associated with a lower level of dialysis-induced cardiac injury. Hemodial Int 13:505-511. 10.1111/j.1542-4758.2009.00384.x

Korsheed S, Eldehni MT, John SG, Fluck RJ, and McIntyre CW. 2011. Effects of arteriovenous fistula formation on arterial stiffness and cardiovascular performance and function. Nephrol Dial Transplant 26:3296-3302. 10.1093/ndt/gfq851 
403 Lee T, Thamer M, Zhang Q, Zhang Y, and Allon M. 2016. Reduced Cardiovascular Mortality

404

405

406

407

408

409

410

411

412

413

414

415

416

417

418

419

420

421

422

423

424

425

426

427

428

429

430

431

432

433

434

435

436

437

438 Associated with Early Vascular Access Placement in Elderly Patients with Chronic Kidney Disease. Am J Nephrol 43:334-340. 10.1159/000446159

Lin MY, Cheng LJ, Chiu YW, Hsieh HM, Wu PH, Lin YT, Wang SL, Jian FX, Hsu CC, Yang SA, Lee HL, and Hwang SJ. 2018. Effect of national pre-ESRD care program on expenditures and mortality in incident dialysis patients: A population-based study. PLoS One 13:e0198387. 10.1371/journal.pone.0198387

London GM, Marchais SJ, Guerin AP, Metivier F, and Pannier B. 1993. Cardiac hypertrophy and arterial alterations in end-stage renal disease: hemodynamic factors. Kidney Int Suppl 41:S42-49.

MacRae JM, Pandeya S, Humen DP, Krivitski N, and Lindsay RM. 2004. Arteriovenous fistulaassociated high-output cardiac failure: a review of mechanisms. Am J Kidney Dis 43:e17-22.

McIntyre CW. 2009. Effects of hemodialysis on cardiac function. Kidney Int 76:371-375. 10.1038/ki.2009.207

Munclinger M, Nemecek K, Serf B, Vondracek V, and Hrudova J. 1987. Effect of arteriovenous fistula creation and maturation on rest hemodynamics in patients with end-stage renal disease. Nephron 46:105-106. 10.1159/000184321

Nakhoul F, Yigla M, Gilman R, Reisner SA, and Abassi Z. 2005. The pathogenesis of pulmonary hypertension in haemodialysis patients via arterio-venous access. Nephrol Dial Transplant 20:1686-1692. 10.1093/ndt/gfh840

National Health Insurance Administration MoHaW, Taiwan, R.O.C. 2014. National Health Insurance Annual Report 2014-2015.122.

Oliver MJ, Rothwell DM, Fung K, Hux JE, and Lok CE. 2004. Late creation of vascular access for hemodialysis and increased risk of sepsis. J Am Soc Nephrol 15:1936-1942.

Ori Y, Korzets A, Katz M, Erman A, Weinstein T, Malachi T, and Gafter U. 2002. The contribution of an arteriovenous access for hemodialysis to left ventricular hypertrophy. Am J Kidney Dis 40:745-752. 10.1053/ajkd.2002.35685

Ori Y, Korzets A, Katz M, Perek Y, Zahavi I, and Gafter U. 1996. Haemodialysis arteriovenous access--a prospective haemodynamic evaluation. Nephrol Dial Transplant 11:94-97.

Ravani P, Palmer SC, Oliver MJ, Quinn RR, MacRae JM, Tai DJ, Pannu NI, Thomas C, Hemmelgarn BR, Craig JC, Manns B, Tonelli M, Strippoli GF, and James MT. 2013. Associations between hemodialysis access type and clinical outcomes: a systematic review. J Am Soc Nephrol 24:465-473. 10.1681/asn.2012070643

Rayner HC, Pisoni RL, Gillespie BW, Goodkin DA, Akiba T, Akizawa T, Saito A, Young EW, and Port FK. 2003. Creation, cannulation and survival of arteriovenous fistulae: data from the Dialysis Outcomes and Practice Patterns Study. Kidney Int 63:323-330. 10.1046/j.1523- 
439

440

441

442

443

444

445

446

447

448

449

450

451

452

453

454

455

456

457

458

459

460

461

462

463

464

1755.2003.00724.x

Reddy YNV, Obokata M, Dean PG, Melenovsky V, Nath KA, and Borlaug BA. 2017. Longterm cardiovascular changes following creation of arteriovenous fistula in patients with end stage renal disease. Eur Heart J 38:1913-1923. 10.1093/eurheartj/ehx045

Robinson BM, Akizawa T, Jager KJ, Kerr PG, Saran R, and Pisoni RL. 2016. Factors affecting outcomes in patients reaching end-stage kidney disease worldwide: differences in access to renal replacement therapy, modality use, and haemodialysis practices. Lancet 388:294-306. $10.1016 / \mathrm{s} 0140-6736(16) 30448-2$

Sandhu JS, Wander GS, Gupta ML, Aulakh BS, Nayyar AK, and Sandhu P. 2004. Hemodynamic effects of arteriovenous fistula in end-stage renal failure. Ren Fail 26:695701.

Savage MT, Ferro CJ, Sassano A, and Tomson CR. 2002. The impact of arteriovenous fistula formation on central hemodynamic pressures in chronic renal failure patients: a prospective study. Am J Kidney Dis 40:753-759. 10.1053/ajkd.2002.35686

Utescu MS, LeBoeuf A, Chbinou N, Desmeules S, Lebel M, and Agharazii M. 2009. The impact of arteriovenous fistulas on aortic stiffness in patients with chronic kidney disease. Nephrol Dial Transplant 24:3441-3446. 10.1093/ndt/gfp276

Vizinho RS, Santos C, Lucas C, Adragao T, and Barata JD. 2014. Effect of the arteriovenous access for hemodialysis on subendocardial viability ratio, pulse pressure and hospitalizations. J Nephrol 27:563-570. 10.1007/s40620-014-0056-1

Wasse H, Speckman RA, and McClellan WM. 2008. Arteriovenous fistula use is associated with lower cardiovascular mortality compared with catheter use among ESRD patients. Semin Dial 21:483-489. 10.1111/j.1525-139X.2008.00467.x

Zoccali C, Tripepi G, and Mallamaci F. 2005. Predictors of cardiovascular death in ESRD. Semin Nephrol 25:358-362. 10.1016/j.semnephrol.2005.05.002 


\section{Figure 1 (on next page)}

Overall flow diagram of the research design and sampling strategy.

AVA: arteriovenous access; HD: hemodialysis; LHID2000: Longitudinal Health Insurance

Database 2000, a validated subgroup extracted from the Taiwan National Health Insurance Research Database. 
8548 Incident HD patients from LHID2000 in 2001-2012

Exclusions:

61 Age less than 20 years at first HD

503 Ever received peritoneal dialysis before first HD year

87 Ever received kidney transplant before first HD year

4624 Not cases of chronic kidney disease before first HD

3507 Eligible individuals

Exclusions:

21 Ever received HD catheter before first HD

339 Received AVA creation less than 30 days before first HD

837 Pre-HD AVA creation

2310 No Pre-HD AVA creation 


\section{Figure 2 (on next page)}

Cumulative incidence of major adverse cardiovascular events in patients with and without prehemodialysis arteriovenous access creation.

MACEs: major adverse cardiovascular events; Pre-HD AVA: prehemodialysis arteriovenous access. 


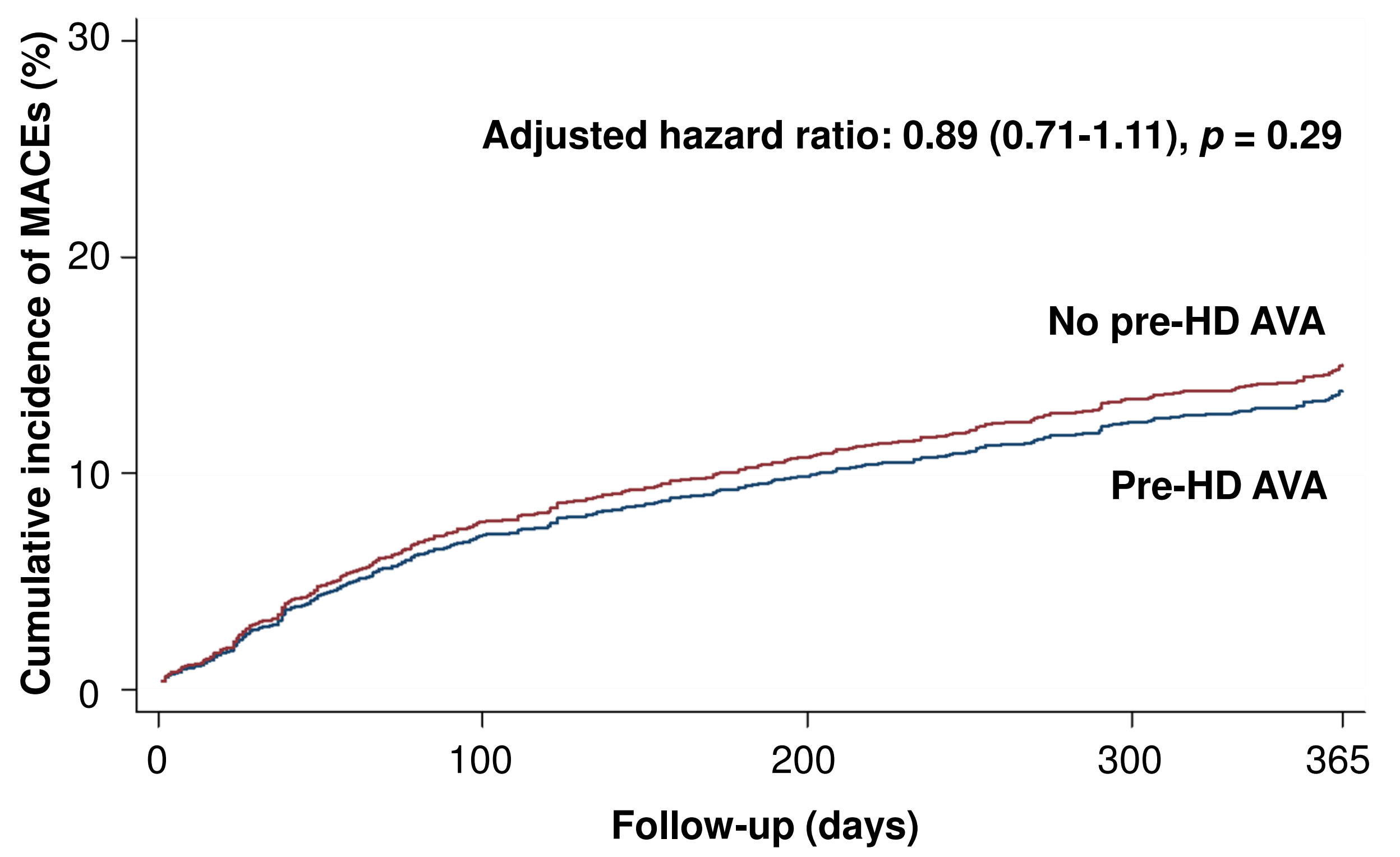




\section{Figure 3 (on next page)}

Cumulative incidence of congestive heart failure in patients with and without prehemodialysis arteriovenous access creation.

CHF: congestive heart failure; Pre-HD AVA: prehemodialysis arteriovenous access. 


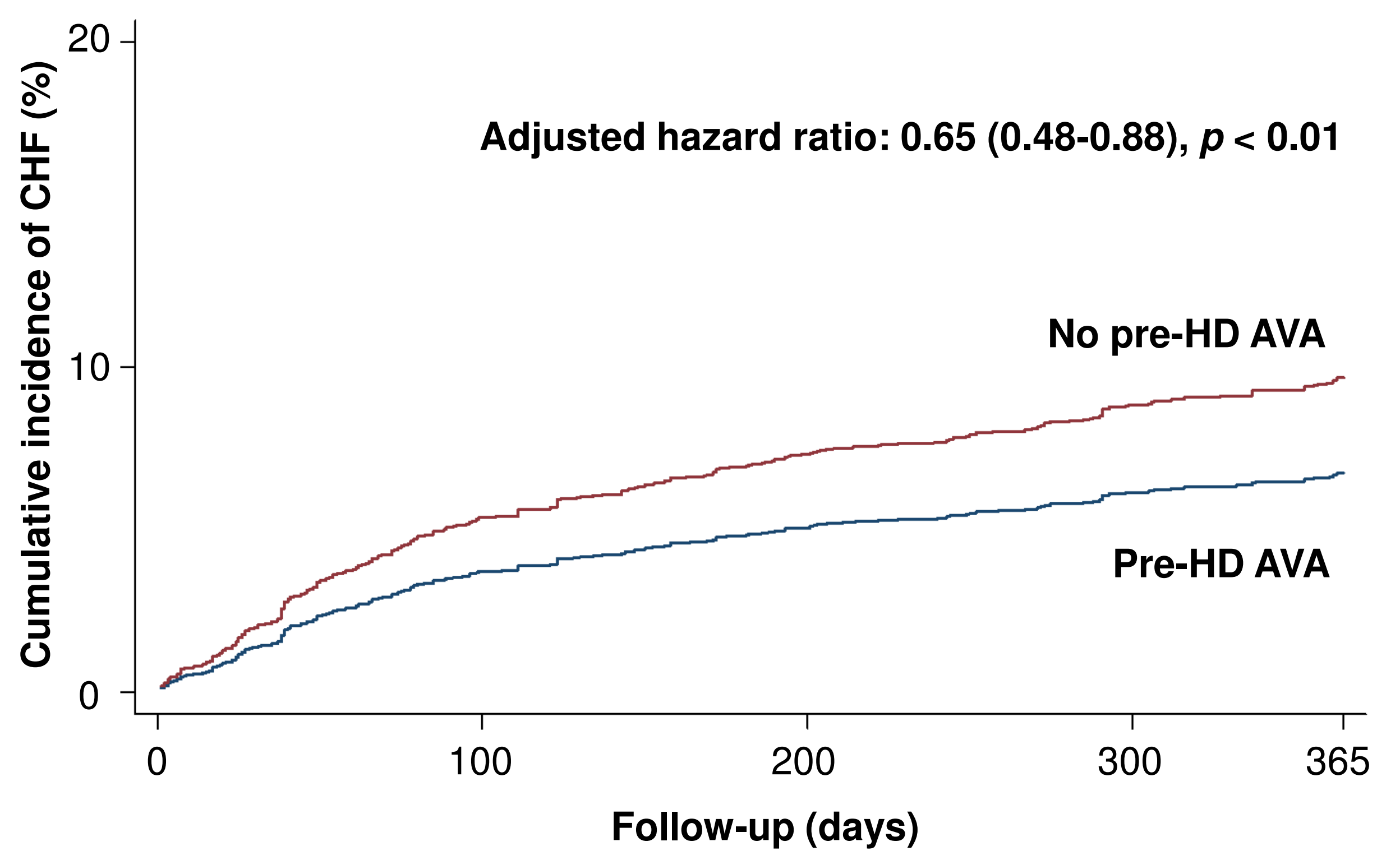




\section{Figure 4 (on next page)}

Cumulative incidence of all-cause mortality in patients with and without prehemodialysis arteriovenous access creation.

ACM: all-cause mortality; Pre-HD AVA: prehemodialysis arteriovenous access. 


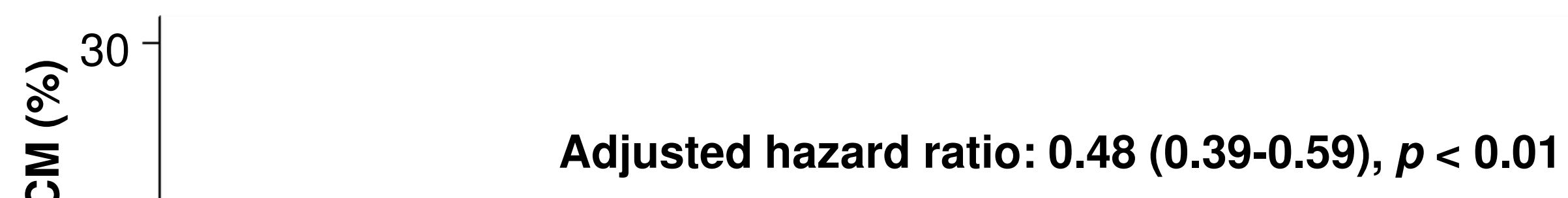

No pre-HD AVA

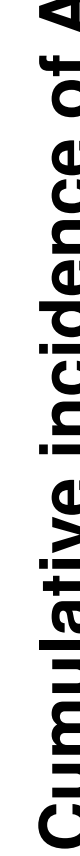

20

10
0
0
0
0
0
0
0
0

$0-$

0

100

200

300

365

Follow-up (days) 


\section{Figure 5(on next page)}

Cumulative incidence of major adverse cardiovascular event-related mortality in patients with and without prehemodialysis arteriovenous access creation.

MACErM: major adverse cardiovascular event-related mortality; Pre-HD AVA: prehemodialysis arteriovenous access. 


\section{Table 1 (on next page)}

Characteristics of patients with and without prehemodialysis arteriovenous access 


\begin{tabular}{|c|c|c|c|c|c|c|}
\hline & Unmatched & & & Matched & & \\
\hline & $\begin{array}{l}\text { Pre-HD AVA } \\
\qquad(\mathrm{N}=837)\end{array}$ & $\begin{array}{l}\text { No pre-HD AVA } \\
\qquad(\mathrm{N}=2310)\end{array}$ & ASD (\%) & $\begin{array}{l}\text { Pre-HD AVA } \\
\qquad(\mathrm{N}=792)\end{array}$ & $\begin{array}{r}\text { No pre-HD AVA } \\
\qquad(\mathrm{N}=792)\end{array}$ & $\operatorname{ASD}(\%)$ \\
\hline \multicolumn{7}{|l|}{ Age, years } \\
\hline Median (IQR) & $66(56-74)$ & $71(59-79)$ & $29.9 *$ & $67(56-75)$ & $66(55-75)$ & 2.42 \\
\hline Sex, male (\%) & $440(52.57)$ & 1157 (50.09) & 4.96 & $401(50.63)$ & $423(53.41)$ & 5.56 \\
\hline Income, NTD/year & & & $15.3^{*}$ & & & 1.83 \\
\hline Dependent & $296(35.36)$ & $878(38.01)$ & & $284(35.86)$ & $288(36.36)$ & \\
\hline $1-19999$ & $206(24.61)$ & $700(30.30)$ & & $197(24.87)$ & $197(24.87)$ & \\
\hline 20000-39999 & $275(32.86)$ & $645(27.92)$ & & $259(32.70)$ & $261(32.95)$ & \\
\hline$\geq 40000$ & $60(07.17)$ & $87(03.77)$ & & $52(06.57)$ & $46(05.81)$ & \\
\hline \multicolumn{7}{|l|}{ Comorbidities } \\
\hline HTN & $712(85.07)$ & $1899(82.21)$ & 7.73 & $670(84.60)$ & $673(84.97)$ & 1.05 \\
\hline IHD & $218(26.05)$ & $649(28.10)$ & 4.61 & $197(24.87)$ & $200(25.25)$ & 0.87 \\
\hline $\mathrm{CHF}$ & $393(46.95)$ & $1150(49.78)$ & 5.66 & $364(45.96)$ & $364(45.96)$ & 0.00 \\
\hline CVA & $76(09.08)$ & $363(15.71)$ & $20.2^{*}$ & $75(09.47)$ & $76(09.60)$ & 0.42 \\
\hline PVD & $45(05.38)$ & $152(06.58)$ & 5.07 & $40(05.05)$ & $47(05.93)$ & 3.88 \\
\hline Dysrhythmia & $50(05.97)$ & $226(09.78)$ & $14.1^{*}$ & 49 (06.19) & $44(05.56)$ & 2.68 \\
\hline DM & $461(55.08)$ & $1303(56.41)$ & 2.60 & $429(54.17)$ & $440(55.56)$ & 2.79 \\
\hline COPD & $81(09.68)$ & $379(16.41)$ & $20.0^{*}$ & $78(09.85)$ & $82(10.35)$ & 1.67 \\
\hline PUD & $186(22.22)$ & $569(24.63)$ & 5.69 & $175(22.10)$ & $172(21.72)$ & 0.91 \\
\hline Liver disease & $65(07.77)$ & $252(10.91)$ & $10.0^{*}$ & $65(08.21)$ & $56(07.07)$ & 4.27 \\
\hline Cancer & $74(08.84)$ & $233(10.09)$ & 4.25 & $71(08.96)$ & $69(08.71)$ & 0.80 \\
\hline Dementia & $20(02.39)$ & $124(05.37)$ & $15.4^{*}$ & $20(02.53)$ & $17(02.15)$ & 2.50 \\
\hline Taiwan index $($ mean $\pm \mathrm{SD})$ & $5.55 \pm 4.00$ & $6.55 \pm 4.40$ & $23.0^{*}$ & $5.52 \pm 4.02$ & $5.52 \pm 4.04$ & 0.09 \\
\hline \multicolumn{7}{|l|}{ Medicine } \\
\hline ESAs & $684(81.72)$ & $804(34.81)$ & $108 .^{*}$ & $639(80.68)$ & $635(80.18)$ & 1.27 \\
\hline Antiplatelets & $537(64.16)$ & $1563(67.66)$ & 7.30 & $505(63.76)$ & $505(63.76)$ & 0.00 \\
\hline Anticoagulants & $75(08.96)$ & $161(06.97)$ & 7.35 & $53(06.69)$ & $51(06.44)$ & 1.00 \\
\hline ACEI / ARBs & $507(60.57)$ & $1579(68.35)$ & $16.3^{*}$ & $485(61.24)$ & $500(63.13)$ & 3.90 \\
\hline Statins & $262(31.30)$ & $665(28.79)$ & 5.48 & $244(30.81)$ & $247(31.19)$ & 0.81 \\
\hline
\end{tabular}

Income was divided into 4 strata according to insurance fees: dependent (patient's medical expenditure was taken charge of the government), $<20000$ New Taiwan Dollars (NTD) per month, $20000-40000$ NTD per month, and $>40000$ NTD per month. The Taiwan index is a weighted comorbidity score of IHD $\times 1+$ CHF $\times 3+$ CVA $\times 4+$ PVD $\times 2+$ COPD $\times 3+$ PUD $\times 2+$ Liver disease $\times$ $4+$ Dysrhythmia $\times 3+$ Cancer $\times 6+\mathrm{DM} \times 3$

Abbreviations: ACEI: angiotensin converting enzyme inhibitor; ARB: angiotensin II receptor blocker; ASD: absolute standard mean difference; CHF: congestive heart failure; COPD: chronic obstructive pulmonary disease; CVA: cerebrovascular accident; DM: diabetes mellitus; ESA: erythropoiesis-stimulating agent; HTN: hypertension; IHD: ischemic heart disease; IQR: interquartile range; Pre-HD AVA: prehemodialysis arteriovenous access; PUD: peptic ulcer disease; PVD: peripheral vascular disease; SD: standard deviation.

$* \mathrm{ASD} \geq 10 \%$ 


\section{Table 2 (on next page)}

Clinical outcomes of patients with and without prehemodialysis arteriovenous access 


\begin{tabular}{|c|c|c|c|c|}
\hline & Unmatched & & Matched & \\
\hline & Crude HR & Adjusted $\mathrm{HR}^{\mathrm{a}}$ & Crude HR & Adjusted $\mathrm{HR}^{\mathrm{b}}$ \\
\hline \multicolumn{5}{|l|}{ Primary outcomes } \\
\hline MACEs & $0.73(0.60-0.89)^{* *}$ & $0.89(0.71-1.11)$ & $0.93(0.73-1.18)$ & $0.94(0.74-1.21)$ \\
\hline $\mathrm{CHF}$ & $0.52(0.40-0.68)^{* * *}$ & $0.65(0.48-0.88)^{* *}$ & $0.63(0.46-0.86)^{* *}$ & $0.63(0.46-0.87)^{* *}$ \\
\hline \multicolumn{5}{|l|}{ Secondary outcomes } \\
\hline All-cause mortality & $0.28(0.23-0.34)^{* * *}$ & $0.48(0.39-0.59)^{* * *}$ & $0.47(0.37-0.60)^{* * *}$ & $0.46(0.36-0.59)^{* * *}$ \\
\hline MACE-related mortality & $0.37(0.25-0.55)^{* * *}$ & $0.70(0.45-1.08)$ & $0.59(0.37-0.93)^{*}$ & $0.60(0.38-0.96)^{*}$ \\
\hline BSI-related mortality & $0.21(0.15-0.30)^{* * *}$ & $0.32(0.22-0.46)^{* * *}$ & $0.30(0.20-0.45)^{* * *}$ & $0.29(0.19-0.44)^{* *}$ \\
\hline
\end{tabular}

aAdjusted for age, sex, income, year of hemodialysis, comorbidities, and medicine; badjusted for age, sex, income, year of hemodialysis, Taiwan index, hypertension, dementia, and medicine. The Taiwan index is a comorbidity index employed for mortality prediction that has been validated for Taiwanese patients undergoing hemodialysis as having adequate reclassification ability. Abbreviations: BSI: bloodstream infection; CHF: congestive heart failure; HR: hazard ratio; MACEs: major adverse cardiovascular events. ${ }^{*} p<0.05 ; * * p<0.01 ; * * * p<.001$ 


\section{Table 3(on next page)}

Sensitivity analyses of clinical outcomes of patients with and without prehemodialysis arteriovenous access 


\begin{tabular}{lllll}
\hline & $\mathrm{SA}^{\mathrm{b}}$ & $\mathrm{SA}^{\mathrm{c}}$ & $\mathrm{SA}^{\mathrm{d}}$ & $\mathrm{SA}^{\mathrm{e}}$ \\
\hline $\begin{array}{l}\text { Primary outcomes } \\
\text { MACEs, aHR }\end{array}$ & & & & \\
$\quad$ CHF, aHR & $0.77(0.62-0.97)^{*}$ & $0.82(0.64-1.05)$ & $0.90(0.74-1.10)$ & $0.88(0.69-1.13)$ \\
Secondary outcomes & $0.57(0.42-0.77)^{* * *}$ & $0.60(0.43-0.84)^{* *}$ & $0.69(0.53-0.91)^{* *}$ & $0.71(0.51-0.98)^{*}$ \\
All-cause mortality, aHR & & & & $0.47(0.36-0.61)^{* * *}$ \\
MACE-related mortality, aHR & $0.94(0.72-1.22)$ & $0.65(0.46-0.91)^{*}$ & $0.50(0.42-0.60)^{* * *}$ & $0.70(0.43-1.17)$ \\
BSI-related mortality, aHR & $0.60(0.39-0.92)^{*}$ & $0.38(0.21-0.67)^{* * *}$ & $0.34(0.25-0.47)^{* * *}$ & $0.29(0.19-0.46)^{* * *}$ \\
\hline
\end{tabular}

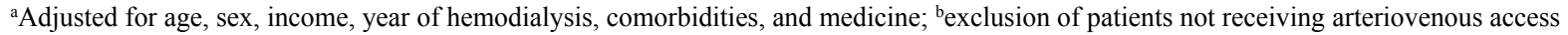

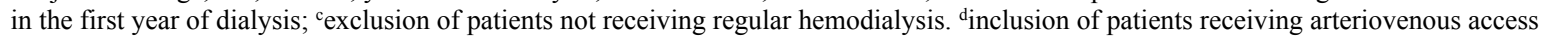
less than 1 month before the first hemodialysis; einverse probability of treatment weighting of study subjects. Abbreviations: aHR: adjusted hazard ratio; BSI: bloodstream infection; CHF: congestive heart failure; MACEs: major adverse cardiovascular events; SA: sensitivity analysis. ${ }^{*} p<0.05 ; * * p<0.01 ; * * * p .001$ 